\title{
Callisto: A Guide to the Origin of the Jupiter System
}

\author{
David E Smith \\ 617-803-3377 \\ Department of Earth, Atmospheric and Planetary Sciences \\ Massachusetts Institute of Technology, Cambridge MA 02139 \\ smithde@mit.edu
}

Co-authors:

Francis Nimmo, UCSC, fnimmo@ucsc.edu

Krishan Khurana, UCLA, kkhurana@igpp.ucla.edu

Catherine L. Johnson, PSI, cjohnson@psi.edu

Mark Wieczorek, OCA, Fr, mark.wieczorek@oca.eu

Maria T. Zuber, MIT, zuber@mit.edu

Carol Paty, University of Oregon, cpaty@uoregon.edu

Antonio Genova, Univ Rome, It, antonio.genova@uniroma1.it

Erwan Mazarico, NASA GSFC, erwan.m.mazarico@nasa.gov

Louise Prockter, LPI, prockter@lpi.usra.edu

Gregory A. Neumann, NASA GSFC Emeritus, gregory.a.neumann@nasa.gov

John E. Connerney, Adnet Systems Inc., john.e.connerney@nasa.gov

Edward B. Bierhaus, LMCO, edward.b.bierhaus@lmco.com

Sander J. Goossens, UMBC, sander.j.goossens@nasa.gov

Michael K. Barker, NASA GSFC, michael.k.barker@nasa.gov

Peter B. James, Baylor, P_James@baylor.edu

James Head, Brown,James_Head@brown.edu

Jason Soderblom, MIT,jms4@mit.edu

July 14,2020 


\section{Introduction}

Among the Galilean moons of Jupiter, it is outermost Callisto that appears to most fully preserve the record of its ancient past. With a surface almost devoid of signs of internal geologic activity, and hints from spacecraft data that its interior has an ocean while being only partially differentiated, Callisto is the most paradoxical of the giant rock-ice worlds. How can a body with such a primordial surface harbor an ocean? If the interior was warm enough to form an ocean, how could a mixed rock and ice interior remain stable? What do the striking differences between geologically unmodified Callisto and its sibling moon Ganymede tell us about the formation of the Galilean moons and the primordial conditions at the time of the formation of Callisto and the accretion of giant planet systems?

The answers can be provided by a Callisto orbital mission.

\section{Why Callisto?}

The ice-rock moons of the outer planets known or suspected to be ocean worlds - Enceladus, Europa, Ganymede, Titan, and Callisto - are key to understanding their host planets' origins and because they may harbor potentially habitable subsurface environments. At Enceladus, Europa, Ganymede, and Titan, widespread geologic activity has erased the record of their initial state. In contrast, Callisto's surface appears untouched by internal processes and hence uniquely preserves the record of its ancient past [e.g., Moore et al., 2004; Zahnle et al., 2003; Zahnle et al., 2001].

Data obtained during Galileo spacecraft flybys hint that Callisto has a sub-surface liquid ocean [Khurana et al., 1998; Kivelson et al., 1999; Zimmer et al., 2000; Jia et al., 2010; Saur et al., 2010], and an underlying mixed ice-rock interior that did not fully differentiate into distinct rock and ice layers [Anderson et al., 2001]. This requires that the ice in this mixture never softened sufficiently to allow the rock to separate out. How a large ice-rock moon can simultaneously produce an ocean and a cold undifferentiated interior is not understood and - if true - will force a re-thinking of how large satellites form and evolve [e.g., Stevenson et al., 1986; Barr \& Canup, 2008, 2010; Monteux et al., 2014].

Figure 1 Callisto is unique in having a preserved interior, an ancient surface, and likely being an ocean world.

However, the existence of an ocean remains uncertain due to the confounding effect of Callisto's time-variable ionosphere on the interpretation of Galileo magnetic field observations that hint at

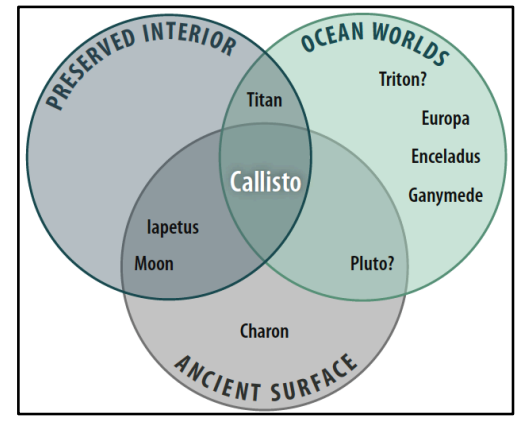
an ocean [Hartkorn \& Saur, 2017a,b]. The inference that Callisto is not fully differentiated is based on the assumption that its interior is hydrostatic, but this assumption is known to be invalid for other moons and expected deviations could instead permit a differentiated interior [e.g., McKinnon 1997, Gao \& Stevenson, 2013].

Callisto - The ancient, enigmatic ice-rock moon: Satellite characteristics can provide crucial constraints on the formation and early history of their host planet, particularly if they were not extensively geologically altered. Notably, studies of our Moon have been central to understanding the timing and nature of Earth's assembly [e.g., Canup 2004]. Callisto, Ganymede and Titan have 
similar sizes and ice-rock content but vary greatly in signs of internal geology and (inferred) interior structures (Figure 2). Their nearly circular, co-planar orbits imply that they formed within circumplanetary disks thought to exist at or near the end of gas giant planet formation; how and when this occurred remains debated [e.g., Lunine \& Stevenson 1982; Canup \& Ward 2002, 2006, 2009; Estrada \& Mosqueira, 2006; Mosqueira \& Estrada 2003a,b; Alibert et al. 2005; Machida et al. 2008; Ward \& Canup 2010; Ogihara et al. 2012; Miguel \& Ida 2016; Cilibrasi et al. 2018].

Figure 2 Schematic of the interior structure of a typical icy moon. The internal structure of Callisto is unknown.

If we could better understand how the large outer moons formed, this would provide critical information on gas planet formation. For instance, an undifferentiated Callisto requires that the moon's growth is complete after shortlived radioactive isotope ${ }^{26} \mathrm{Al}$ is exhausted $(>4 \mathrm{Myr}$ after solar system formation), the growth timescale is long $(>0.6$ Myr), and that the protosatellite disk was cold [Barr and Canup, 2008].

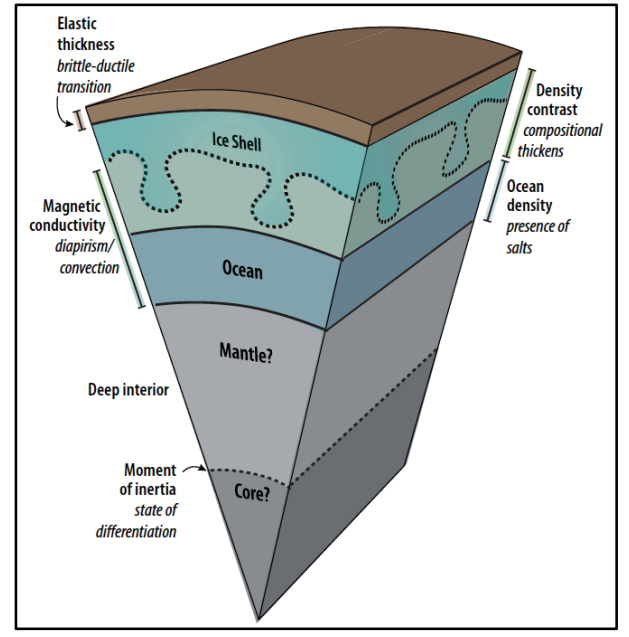

Each of these criteria informs gas giant formation: Jupiter formation cannot have been complete before $4 \mathrm{Myr}$, while Callisto's growth timescale and disk temperature depend on the disk surface density and viscosity, respectively, both of which control how rapidly solids are delivered to Jupiter [Lunine \& Stevenson 1982]. The delivery rate of solid material in turn controls when Jupiter can start to acquire significant gas. Thus, as emphasized in the last Decadal Survey, the outer satellites may thus retain "geological records of the processes of formation and evolution in the outer solar system -records no longer accessible in the giant planets themselves."

By virtue of its ancient state, Callisto is best able to inform how the Jovian satellites formed and evolved. First, Callisto's cratered surface preserves the record of its early geologic history, including giant collisions that produced enormous multi-ring basins. Studies of lunar basins [e.g., Melosh et al., 2013; Neumann et al., 2015; Johnson et al., 2016] using GRAIL gravity [Zuber et al., 2013] and Lunar Reconnaissance Orbiter LOLA topography [Smith et al., 2016] data applied at Callisto will constrain its early ice shell thickness and thermal state [e.g., Melosh \& McKinnon, 1978; McKinnon \& Melosh, 1980]; information that is not available at other ocean worlds.

Second, Callisto is inferred to have a vastly different interior structure than neighboring Ganymede, which is a mystery because they formed adjacent in the Jovian disk. The moment of inertia (MOI) factor derived from Galileo flyby data implies Ganymede is fully differentiated $\left(\mathrm{C} / \mathrm{MR}^{2}=0.3105 \pm 0.0028\right.$, Anderson et al., 1996). In contrast, Galileo data combined with the hydrostatic assumption are interpreted as implying that Callisto is only partially differentiated $\left(\mathrm{C} / \mathrm{MR}^{2}=0.355 \pm 0.005\right.$, Anderson et al., 2001), which requires a very restrictive set of formation and evolution conditions. When ice softens and melts, entrained higher-density rock rapidly descends [e.g., Friedson \& Stevenson, 1983]. If differentiation occurs, it is irreversible.

\section{What do we need to know and how well do we need to know it?}

We need to answer the following four questions: 


\section{Is Callisto differentiated?}

Answer: Measure moment of inertia, state of hydrostatic equilibrium, thickness of the ice shell and its density, gravity and body tides, and the low-degree gravity field.

\section{Does Callisto have an ocean?}

Answer: Measure magnetic induction, depth of the conducting layer, and the thickness and density of any ocean.

\section{Has Callisto evolved?}

Answer: Measure obliquity, tides and the acceleration of Callisto in its orbit due to tidal resonance-locking with the interior of Jupiter.

\section{What is the relative age(s) of the ice shell?}

Answer: Measure the bombardment history and structure of the ice shell including relative regional age(s), global shape, crater and basin topography, crater density and morphology, and high-resolution gravity.

Figure 3 Obtaining density from gravity and admittance; indicating the need for gravity resolution to be greater than

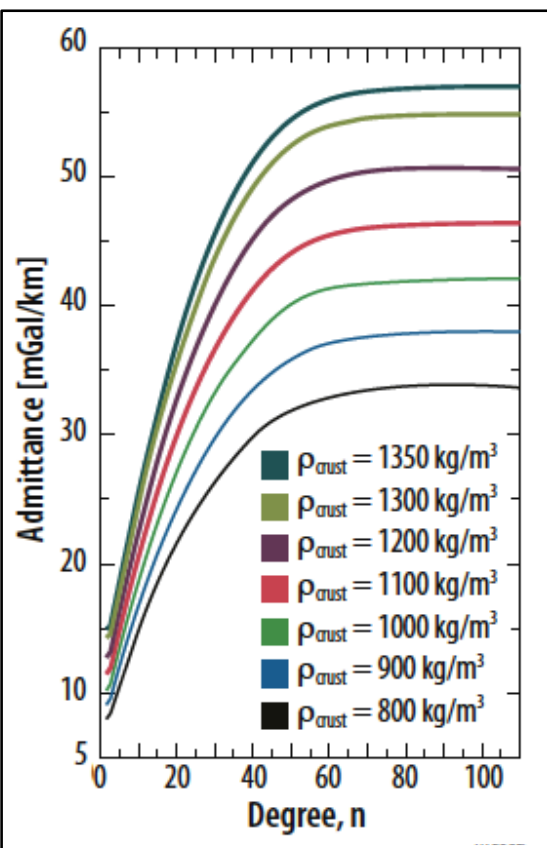
$l=80$. ( " $\mathrm{n}$ " is the spherical harmonic degree of the gravity field model).

The adequacy of these datasets for providing the models of Callisto needed to understand its origin and evolution has been demonstrated in a simulation of a year-long, low-altitude orbital mission of Callisto that would carry previously flown instrumentation for topography, magnetometry, imagery and would conduct a gravity investigation. Table 1 summarizes the uncertainties required for the primary parameters of gravity, tidal Love Numbers, shape/topography, image resolution and coverage, magnetic induction, Callisto's obliquity and orbital acceleration.

\begin{tabular}{|l|l|}
\hline Gravity field & Spherical harmonic degree \& order $\geq 90$ (spatial blocksize $84 \mathrm{~km}) ; l=2 \pm 0.1 \%$ \\
\hline Magnetic field & $\begin{array}{l}\text { Depth to induction layer: } 5 \mathrm{~km} \text {; induced field intensity } \pm 1 \mathrm{nT}(10-\mathrm{hr} \text { signal), } \\
\pm 0.5 \mathrm{nT}\left(400 \text {-hr signal), induced phase lag } \pm 5^{\circ}\right.\end{array}$ \\
\hline Tides & $\mathrm{k}_{2}: \pm 0.1 \% ; \mathrm{h}_{2}: \pm 0.5 \%$ \\
\hline Topography & $\begin{array}{l}\text { Shape: } \pm 5 \mathrm{~m} \text {; topography: } \pm 1 \mathrm{~m} \text { (MOI \& SOE); topography: } \pm 10 \mathrm{~m} \text {, profiles } \pm 5 \\
\mathrm{~m} \text { (shell structure) }\end{array}$ \\
\hline Imaging & $\begin{array}{l}\text { All craters }>5 \text {-km diam (morphology); craters }>2 \mathrm{~km} \text { (freq.-size distribution); } \\
50 \text { - } \mathrm{m} / \mathrm{px} 95 \% \text { of surface }\end{array}$ \\
\hline Callisto pole & RA: $\pm 10^{-3} \mathrm{deg} ;$ Dec: $\pm 10^{-3} \mathrm{deg}$ \\
\hline Callisto orbit & Obliquity: $\pm 0.01^{\circ}$, orbit position: $\pm 5 \mathrm{~m}$ along tr., $\pm 1 \mathrm{~m}$ radial \\
\hline
\end{tabular}

Table 1 Primary observations and required accuracies to resolve the structure of Callisto from a study of a Callisto orbiter mission.

High resolution data of the kind in Table 1 have been demonstrated by the Lunar Reconnaissance Orbiter (LRO) and GRAIL missions to the Moon and acquired some of the highest-resolution imagery, gravity and topography of any planetary body over a period of 1 Earth year. The addition 
of magnetometer measurements of the kind presently being obtained on the Juno mission would complete the primary measurement set.

\section{Present knowledge base}

The existence of an ocean at Callisto is less well established than at Europa, because the magnetic signal detected by Galileo could also be explained by induction in a highly-conducting ionosphere [Hartkorn \& Saur, 2017b]. However, no significant ionosphere was detected on one Galileo flyby (C9), but there was a strong signature of induction [Kliore et al., 2002]. Because the presence of an ocean imposes critical constraints on Callisto's formation and evolution [e.g., Nagel et al., 2004; McKinnon, 2006], it is imperative that the detection of the ocean not rely on this single flyby.

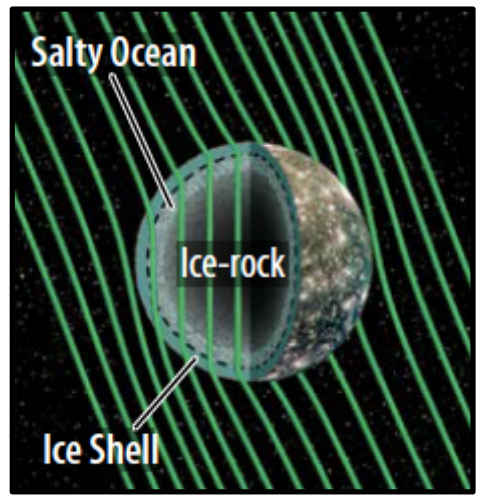

Figure 4: Callisto and Jupiter's magnetic field

In contrast, five Galileo flybys provided data on the degree-2 gravity coefficients which indicated a large MOI and hence an incomplete state of differentiation. However, this determination of the MOI rested on the assumption that the interior is in hydrostatic equilibrium as Galileo measurements did not allow for independent measurement of Callisto's $\mathrm{J}_{2}$ and $\mathrm{C}_{22}$ gravity coefficients and hence the extent of non-hydrostaticity. It is probable that non-hydrostatic effects will be significant at Callisto, perhaps even more than at Titan [Iess et al., 2010; Durante et al., 2019] and Ganymede [Palguta et al., 2006], where small deviations are observed. In addition, Callisto is a slowly rotating body, so even modest internal mass variations will influence its MOI significantly [Mueller \& McKinnon, 1988; McKinnon, 1997]. If Callisto is significantly nonhydrostatic, the MOI derived under the hydrostatic equilibrium assumption would likely be too large [Gao \& Stevenson, 2013], possibly invalidating the conclusion that the interior is only partly differentiated. Even in the presence of modestly non-hydrostatic effects, the moment of inertia can still be recovered if both gravity and topography are known, as at Enceladus [Iess et al., 2014].

\section{Expectations of approved future missions}

Two major flight missions are in development phase for Jupiter's ice moons; the ESA JUICE mission to Ganymede, and the NASA Clipper mission to Europa; both of which will use flybys of Callisto to reduce their encounter velocities. At the present time the details of Clipper's flybys of Callisto are unknown.

ESA's JUICE mission will begin a tour of the Jovian system in early 2030; the current mission design includes 10 to 12 Callisto flybys en route to Ganymede orbit insertion. During the flybys JUICE expects to acquire gravity, altimetry and magnetic field data to contribute to the understanding of Callisto and its interior.

The contribution that JUICE can make is dependent upon the flyby altitudes and coverage of Callisto. Based upon the released JUICE flyby trajectories it is possible to assess their contribution to Callisto's gravity, altimetry, and ocean detection. JUICE's contribution to gravity and tides $\left(\mathrm{k}_{2}\right)$ has been estimated based upon a simulation of the published flyby trajectories and compared to present knowledge, and an orbiter in a 50-km altitude orbit for 1-Earth-year. Results are shown in Tables $2 \& 3$. 


\begin{tabular}{|c|c|c|c|c|}
\hline \multirow{2}{*}{$\begin{array}{c}\text { Gravity } \\
\text { Coefficient }\end{array}$} & \multicolumn{2}{|c|}{ Anderson et al., 2001} & \multirow{2}{*}{$\begin{array}{l}\text { Predicted JUICE } \\
\text { Uncertainty }\end{array}$} & \multirow{2}{*}{$\begin{array}{l}\text { Predicted Orbiter } \\
\text { Uncertainty }\end{array}$} \\
\hline & Value & Uncertainty & & \\
\hline $\mathrm{J}_{2}\left(-\mathrm{C}_{20}\right)$ & $-1.46 \times 10^{-5}$ & $3.58 \times 10^{-7}$ & $1.9 \times 10^{-7}$ & $1.4 \times 10^{-10}$ \\
\hline $\mathrm{C}_{22}$ & $1.58 \times 10^{-5}$ & $4.65 \times 10^{-7}$ & $1.1 \times 10^{-7}$ & $1.4 \times 10^{-10}$ \\
\hline Correlation & 0.997 & - & $0.3-0.5$ & 0.05 \\
\hline
\end{tabular}

Table 2. Comparison of JUICE and an orbiter's gravity performance. An orbiter is able to improve on the uncertainties by 3 orders of magnitude compared to JUICE. Also, low correlation between the coefficients is essential for the independence of the results and robust estimation of the MOI and SOE (state of hydrostatic equilibrium). The very large correlation from the Galileo mission is the main reason for doubt about the present state of Callisto's state of differentiation and also for the not-insignificant correlation expected from JUICE.

\begin{tabular}{|c|c|c|c|c|}
\hline Tide & $\begin{array}{l}\text { Expected } \\
\text { value }\end{array}$ & $\begin{array}{l}\text { Predicted JUICE } \\
\text { Uncertainty }\end{array}$ & $\begin{array}{l}\text { Predicted Orbiter } \\
\text { Uncertainty }\end{array}$ & $\begin{array}{c}\text { Shell Thickness from Orbiter } \\
\mathbf{k}_{2}, \mathbf{h}_{2} \text { [Wahr et al., 2006] }\end{array}$ \\
\hline $\mathrm{k}_{2}$ & $\sim 0.4$ & 0.07 & $1.4 \times 10^{-4}$ & \multirow{4}{*}{ 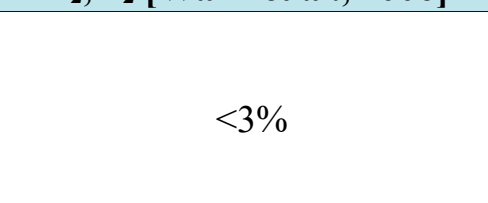 } \\
\hline $\mathrm{k}_{2}$, phase & $?$ & - & $0.03^{\circ}$ & \\
\hline $\mathrm{h}_{2}$ & $\sim 1.2$ & - & $2.0 \times 10^{-3}$ & \\
\hline $\mathrm{h}_{2}$, phase & $?$ & - & $0.2^{\circ}$ & \\
\hline
\end{tabular}

Table 3. Tidal amplitude and phase recovery from 12 simulated JUICE flybys and from a 1-year orbiter mission. Shell elastic thickness assumes ice shell is $>100 \mathrm{~km}$ thick.

If Callisto is non-hydrostatic, determining its MOI and degree of differentiation will require, in addition to the degree- 2 gravity coefficients, the topographic equivalents of $\mathrm{J}_{2}$ and $\mathrm{C}_{22}$ from topography, which are not obtainable by JUICE flybys to required accuracy (Table 1). Although local topography is expected to be possible during flybys a global shape model will be essentially impossible to the required accuracy due to the limited flyby coverage in latitude and longitude and potential trajectory uncertainty.

Magnetic induction observations by JUICE are unlikely due to the signal strength from the altitudes of the flybys ( 200 to $800 \mathrm{~km}$ ), the likely presence of an ionosphere, and the limited duration of the individual flybys. The need for a long time series of magnetic observations has been demonstrated in a simulation of a year-long orbital mission (Figure 5).

Figure 5 Jupiter's magnetodisc is tilted to Callisto's orbital plane, creating a time-varying signal within Callisto's induced magnetic field at Jupiter's rotation period $(10 \mathrm{hr})$. Callisto's orbit around Jupiter is $\sim 400 \mathrm{hr}$, creating another peak in the magnetic response. The ionosphere introduces

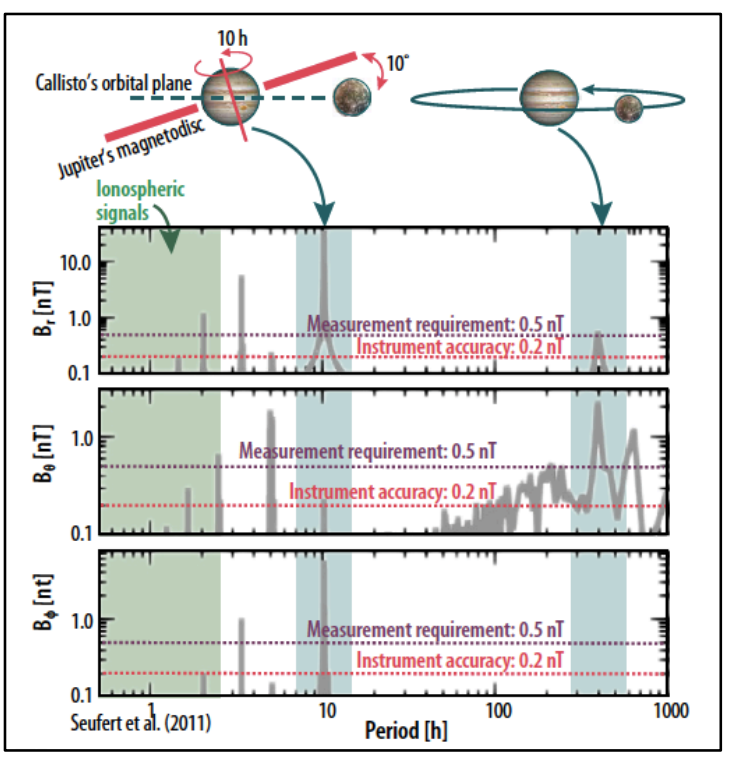
noise with periods of hours and becomes unimportant as the length of the dataset increases, enabling the amplitude at periods of interest to be observed.

\section{Confirming any Callisto orbital acceleration due to tidal dissipation in Jupiter's interior.}


Callisto's orbital evolution is significantly affected by the tidal dissipation in Jupiter's interior [Fuller et al. 2016; Lainey et al. 2020] and is predicted to have a present outward migration rate of order $0.8 \mathrm{~m} /$ year, equivalent to an offset in the along-track position of $165 \mathrm{~m}$ over 1 year. Tracking of an orbiting spacecraft by both range and range-rate for orbit determination analysis is fundamental to estimate both Jupiter's system barycenter and Callisto's orbital dynamics and provide an estimate of its acceleration. The simulated mission estimated the spacecraft orbit, and the initial state for Callisto, which were co-integrated continuously through the 1-year mission (Table 4). This measurement is likely impossible for any other Galilean moon due to their orbital synchronization.

Table 4 Uncertainties in physical parameters of Callisto and its orbit. Note the very accurate estimate of Callisto's obliquity, pole position and along-track position of Callisto from an orbiter mission. These measurements cannot be made from the limited number of JUICE flybys.

Other parameters estimated in the simulation included: Callisto's gravity field and orientation; Callisto's Love number $k_{2}$ and phase lag; GM of Jupiter, Io, Europa and Ganymede; and Jupiter's Love number $k_{2}$ and phase lag,

\begin{tabular}{|lcc|}
\hline Parameter & $\begin{array}{c}\text { JUICE } \\
\text { Flybys } \\
12\end{array}$ & $\begin{array}{c}\text { Orbiter } \\
\mathbf{5 0 ~ k m ~ a l t ~} \\
\text { after 1 yr }\end{array}$ \\
\hline $\begin{array}{l}\text { Max grav. field } \\
\text { (deg \& order) }\end{array}$ & 7 & 100 \\
RA pole, deg & - & $5.8 \times 10^{-5}$ \\
Dec pole, deg & - & $7.0 \times 10^{-5}$ \\
$\begin{array}{l}\text { Obliquity, deg } \\
\text { Callisto, along } \\
\text { track position }\end{array}$ & - & $\mathbf{1 . 0} \times \mathbf{1 0}^{-4}$ \\
$\begin{array}{l}\text { Callisto, radial } \\
\text { position }\end{array}$ & - & $\mathbf{2 ~ m}$ \\
Mean radius, km & 1 & 0.005 \\
\hline
\end{tabular}
which measures its tidal dissipation (Table 3). Table 4 shows the retrieved accuracy of Callisto's position obtained by propagating the formal uncertainties of Callisto's initial condition through the end of the mission. These attainable accuracies demonstrate that the perturbation of Callisto's ephemeris induced by Jupiter's tidal dissipation is achievable. The retrieved uncertainty of Jupiter's phase lag is $0.03 \mathrm{deg}$ (Table 3). Measuring Callisto's tidal response would also provide information on the thickness and temperature structure of the ice shell (Moore \& Schubert 2003).

\section{Summary}

In this white paper we argue that a mission to orbit Callisto can be expected to resolve most of the primary issues regarding Callisto's present-day structure, and the conditions in the Jovian system at the time of formation. With an expected capability of making $\sim 3$ orders of magnitude improvement over any planned future mission for parameters that define the interior, including the state of differentiation, and hydrostatic state, an orbital mission would also answer the questions about a possible ocean and its depth. Further, current technology and existing flight instrumentation all appear adequate for achieving the desired results, which are not possible from a multiple flyby mission. Beyond a full description of any ocean, an orbiter would also provide similar "ground-breaking" information for Callisto that the GRAIL and LRO missions have provided for the Earth's Moon. Further, a Callisto mission together with the Flagship Class missions to Europa and Ganymede offers a unique opportunity to understand the observed differences between these ocean worlds and the conditions at the time of their formation.

\section{References}

Alibert, Y., et al. (2005) I. Astron. Astrophys. 439, 1205-1213.

Anderson, J.D., et al. (1996) Nature 384, 541-543.

Anderson, J.D., et al. (2001) Icarus 153, 157-161.

Barr, A.C. and R.M. Canup (2008) Icarus 198, 163-177. 
Barr, A.C. and R.M. Canup (2010) Nature Geoscience, 3, 164-167.

Canup, R.M. and W.R. Ward (2002) Astron. J., 124, 3404-3423.

Canup, R.M. and W.R. Ward (2006) Nature, 441 834-839.

Canup, R.M. and W.R. Ward (2009) In Europa, Univ. Az. Press, pp. 59-84.

Cilibrasi, M. and J. Szulagyi (2008) Mon. Not. Royal Astron. Soc. 480, 4355- 4368.

Durante, D., et al. (2019) Icarus 326, 123-132.

Estrada, P.R. and I. Mosqueira, (2006) Icarus, 181, 486-509

Friedson, A.J. and D.J. Stevenson (1983) Icarus 46, 1-14.

Fuller J., et al. (2016) Mon. Not. R. Astron. Soc. 458, 3867-3879.

Gao, P. and D.J. Stevenson (2013) Icarus 226,1183-1191.

Hartkorn O. and J. Saur (2017a) Icarus 282, 237-259.

Hartkorn, O and J. Saur (2017b) J. Geophys. Res. -- Space Phys. 122, 11,677-11,697.

Iess, L., Rappaport, et al. (2010) Science 327, 1367-1369.

Iess, L., et al. (2014) Science 344, 78-80.

Jia, X., et al. (2010) Space Sci. Rev. 152,271-305.

Khurana, K.K., et al. (1998) Nature 395, 777-780.

Kivelson, et al. (1999) J. Geophys. Res. 104, 4609-4625.

Kliore, A.J., et al., (2002) J. Geophys. Res., 107(A11), doi: 10.1029/2002JA00936.

Lainey, V., et al., Nat Astron (2020). https://doi.org/10.1038/s41550-020-1120-5

Lunine, J.L. and D.J. Stevenson (1982) Icarus 52,14-39.

Machida, M.N., et al., (2008) Astrophys. J. 685, 120-1236

McKinnon W.B. and HJ.Melosh(1980). Icarus 44, 454-471.

McKinnon, W.B. (2006) Icarus 183, 435-450.

Melosh, H.J. and W.B. McKinnon (1978) Geophys. Res. Lett. 5, 985-988.

Miguel, Y. and S. Ida (2016) Icarus 266, 1-14.

Monteux, J., et al. (2014). Icarus 237, 377-387.

Moore W.B. and G. Schubert (2003) Icarus 166, 223-226.

Mosqueira, I and P.R. Estrada (2003a) Icarus 163, 198-231, (2003b) Icarus 163, 232-255

Moore J.M., et al. (2004) Callisto, pp. 397-426, in Jupiter, Cambridge Univ. Press.

Mueller, S. and W.B. McKinnon (1988) Icarus 76, 437-464.

Nagel, K., et al. (2004) Icarus 169,402-412.

Ogihara, M. and S. Ida (2012) Astrophys. J. 753:60, 70 pp.

Olsen, N., et al. (2010) Space Sci. Rev. 152, 135-137.

Palguta, J., et al. (2006) Icarus 180, 428-441.

Saur J., et al. (2010) Space Sci. Rev. 152, 391-421.

Smith, D.E., et al. (2016) Icarus 283, doi: 10.1016/j.icarus.2016.06.006.

Stevenson, D.J., et al. (1986) Origin of satellites. Univ. Arizona Press, pp. 39-88.

Wahr, J.M., et al. (2006) Journal Geophys. Res. 111, E12005.

Ward, W.R. and R.M. Canup (2010) Astron. J. 140, 1168-1193.

Zahnle, K., et al. (2003) Icarus 163, Issue 2, 263-289.

Zahnle, K., et al. (2001) Icarus 153, Issue 1, 111-129.

Zimmer, C., et al. (2000) Icarus 147, 329-347.

Zuber, M.T. et al. (2013) Science 399, doi: 10.1126/science.1231530. 